Session E

Oscillations, mass loss and convection 


\title{
Application of convection simulations to oscillation excitation and local helioseismology
}

\author{
Robert F. Stein ${ }^{1}$, David Benson ${ }^{1}$, Dali Georgobiani ${ }^{1}$ \\ and Åke Nordlund ${ }^{2}$ \\ ${ }^{1}$ Department of Physics and Astronomy, Michigan State University, East Lansing, MI 48824, \\ USA \\ email: stein@pa.msu.edu \\ ${ }^{2}$ Niels Bohr Institute, Copenhagen University, Juliane Maries Vej 30, DK-2100, København Ø, \\ DK \\ email: aake@astro.ku.dk
}

\begin{abstract}
Excitation of f- and p- modes by Reynolds stresses and entropy fluctuations is reviewed. Approximations made to allow semi-analytic analysis are discussed. The spectrum of solar convection is presented and shown to NOT be separable into independent spatial and temporal factors. An appropriate fitting formula is presented.

Time-distance local helioseismology has been analyzed using numerical simulations. One approach is simulation of solar surface convection on supergranule scales (48 Mm wide by $20 \mathrm{Mm}$ deep). A surface shear layer develops. There is a continuous increase in the horizontal scale of the convective motions with increasing depth. Some small granular scale downflows at the surface are swept sideways by diverging larger scale upflows from below to merge into stronger downdrafts in these larger downflow boundaries. Elsewhere, some granular downflows have to beat their way against the upflows from below are halted. These simulations have a rich spectrum of $\mathrm{f}$ - and p- modes that turn within the computational domain. Cross-correlations between each surface location and each location below the surface reveals the wave propagation pattern from the surface. Waves at the surface that propagate into the interior, spread horizontally and are refracted back toward the surface. Time-distance diagrams have been constructed and inverted to determine the subsurface flows, which can then be compared with the average flows in the simulation.
\end{abstract}

A second approach calculates the propagation of linearized waves through a fixed, but nonuniform, background state. Some examples of such analysis are presented.

Keywords. Convection, Sun:oscillations, Sun:helioseismology

\section{Wave excitation}

Our reasons to make numerical simulations of solar convection is to understand physical processes, to validate methods used to analyze observations (particularly those of local helioseismology) and to understand the observations.

One application of realistic solar convection simulations is to understand the mechanism of and calculate the rate of excitation of stellar $p$-mode oscillations. The excitation mechanism for solar oscillations is the $P d V$ work of the turbulent pressure (or Reynolds stresses) and the non-adiabatic gas pressure fluctuations (which are equivalent to the 
entropy fluctuations). The excitation rate is

$$
\frac{\Delta\left\langle E_{\omega}\right\rangle}{\Delta t}=\frac{\omega^{2}\left|\int_{r} d r \delta P_{\omega}^{*} \frac{\partial \xi_{\omega}}{\partial r}\right|^{2}}{8 \Delta \nu E_{\omega}}
$$

Here, $\delta P_{\omega}^{*}$ is the discrete Fourier amplitude of the pseudo-Lagrangian variation of the incoherent turbulent pressure and non-adiabatic gas pressure; $\xi_{\omega}(r)$ is the radial p-mode of angular frequency $\omega$ displacement eigenfunction and $\partial \xi_{\omega} / \partial r$ is the mode compression; $E_{\omega}$ is the mode energy; $\Delta \nu$ is the reciprocal of the duration over which the excitation is evaluated (see Nordlund \& Stein 2001, Stein \& Nordlund 2001 and Stein et al. 2004 for details). This expression can be evaluated using data from convection simulations.

In much of the analytic work on oscillation excitation (e.g. Goldreich et al. 1994, Samadi \& Goupil 2001) it was assumed that the mode compression factor was slowly varying and could be pulled outside the integral giving an excitation rate that was the product of the sum of the squares of the turbulent and gas pressure fluctuations times the square of the mode compression. This eliminates the interaction between the two different pressure contributions and the effect of spatially varying compression. That is not necessary and Houdek has now dropped this simplifying assumption. In fact, the turbulent pressure and entropy fluctuation contributions to the driving sometimes reinforce each other and sometimes interfere destructively. There is also some cancellation between excitation close to the surface and damping at larger depths.

The excitation spectrum decreases at low frequencies because of mode properties - the mode mass increases toward low frequencies and the mode compression decreases toward low frequencies. The excitation spectrum decreases toward high frequencies because of the properties of the convection - the pressure fluctuations decrease at high frequencies because convection is a low frequency phenomenon.

Another assumption made in analytic excitation expressions is that the turbulence spectrum can be factored into spatial and temporal factors. Based on our hydrodynamic convection simulations we find that the turbulent energy spectrum can't be separated into independent spatial and temporal factors. The temporal spectrum depends on the spatial wave number and the spatial spectrum depends on the frequency. The spectrum can be fit by a formula of the form

$$
E(k, \nu)=E(k)\left[\nu^{2}+w(k)^{2}\right]^{-n(k)}
$$

where width $w(k)$ and the exponent $n(k)$ are functions of the spatial scale.

The relative importance of Reynolds stresses and entropy fluctuations has also been discussed with differing conclusions (Goldreich et al. 1994, Stein \& Nordlund 2001, Samadi et al. 2001, Samadi et al. 2003). Entropy fluctuations are significant near optical depth unity where energy transport is changing from convective to radiative. Locally in space and time they are not in balance, so that there is local heating and cooling which produces entropy fluctuations. Near the surface the work of the non-adiabatic gas pressure (entropy) fluctuations is comparable to the work of the turbulent pressure (Reynolds stress) fluctuations. But entropy fluctuations are only important near the surface. Turbulent pressure fluctuations, on the other hand, are large where the convective velocities are large, which occurs over a much deeper range, especially at low frequencies. For the Sun, and most of the other stars we have looked at, the dominant excitation mechanism is the turbulent pressure fluctuations. 


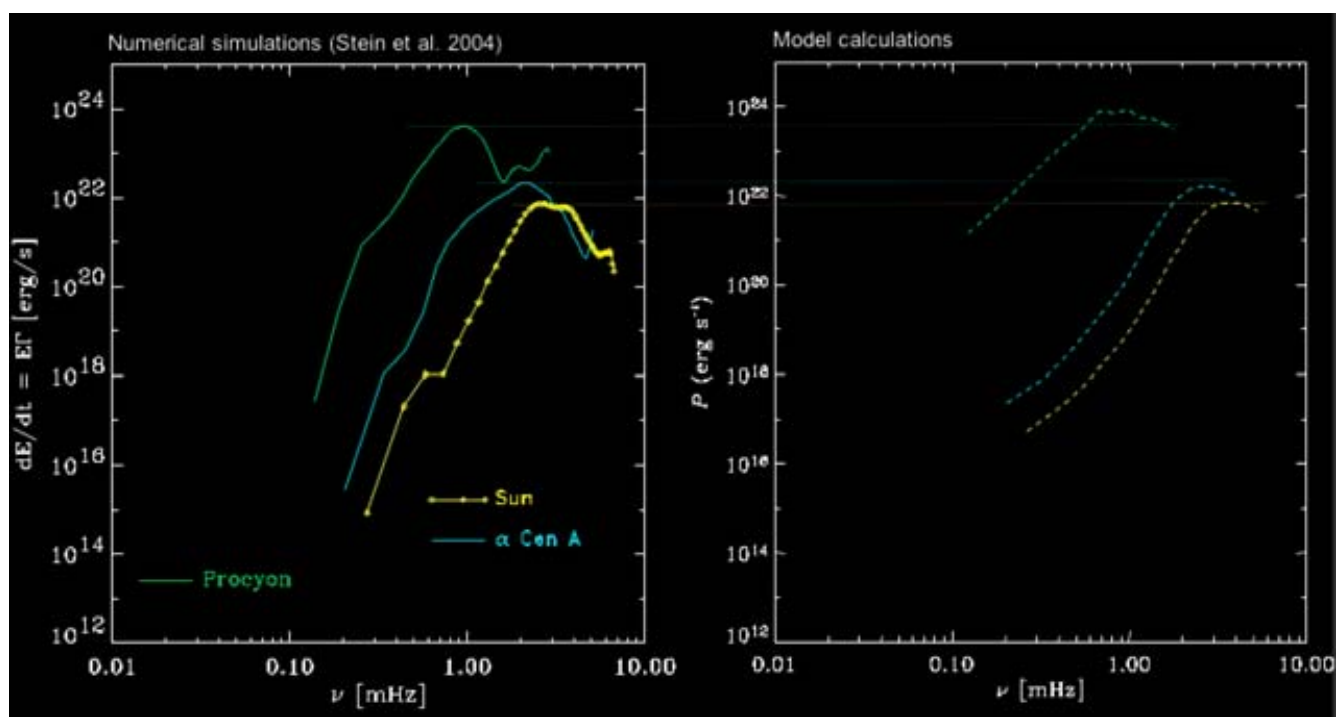

Figure 1. Comparison of numerical and analytic $p$-mode excitation rates for three stars: the Sun, $\alpha$ Cen A and Procyon (upper curve) using Houdek's analytic expression.

Günter Houdek has developed an analytic theory of mode excitation that does not separate the pressure fluctuations from the mode compression terms,

$$
P=\frac{\pi}{9 I}(\Phi \Psi)^{2} \int_{0}^{R}\left(r p_{t} \frac{\partial \xi}{\partial r}\right)^{2} \ell^{3} S(r, \omega) d r
$$

This is a big improvement over past theories. He uses a mixing-length convective model and does not included the driving by entropy fluctuations. He has compared his excitation results with our numerical results for several stars (Fig. 1). The mixing-length convective model leads to a narrower layer of large turbulent pressure with a larger maximum ratio of turbulent to gas pressure.

\section{Supergranulation scale convection simulation}

Another important application of realistic convection simulations is to test and refine local helioseismic inversion methods. There has been no validation of local helioseismology inversion methods to date. Our goal is to provide a data set that the local helioseismologists can apply their inversion techniques to and compare their results with what is actually happening in the simulation. In order for them to do that they need a large size because they are looking at waves that propagate down into the interior and return to the surface in a different place. The deeper the waves penetrate into the interior the farther away they return to the surface. A long time sequence is needed to separate the signal from the noise. We have a simulation that is $48 \mathrm{Mm}$ wide by $20 \mathrm{Mm}$ deep that has run for 65 hours of solar time. This is supergranulation scale, so a bonus is that we will learn something about the nature of supergranulation. Our intention is to double the horizontal size to $96 \mathrm{Mm}$ wide, which is what people doing ring diagram analysis tell us they need and will also permit time-distance analysis down to depths of order $10 \mathrm{Mm}$.

The turnover time at $20 \mathrm{Mm}$ depth is about 48 hours. In order to relax the simulation both thermally and dynamically we needed to run it for many turnover times. We didn't run it for many turnover times at the full horizontal scale. We started at $12 \mathrm{Mm}$ wide for 4 

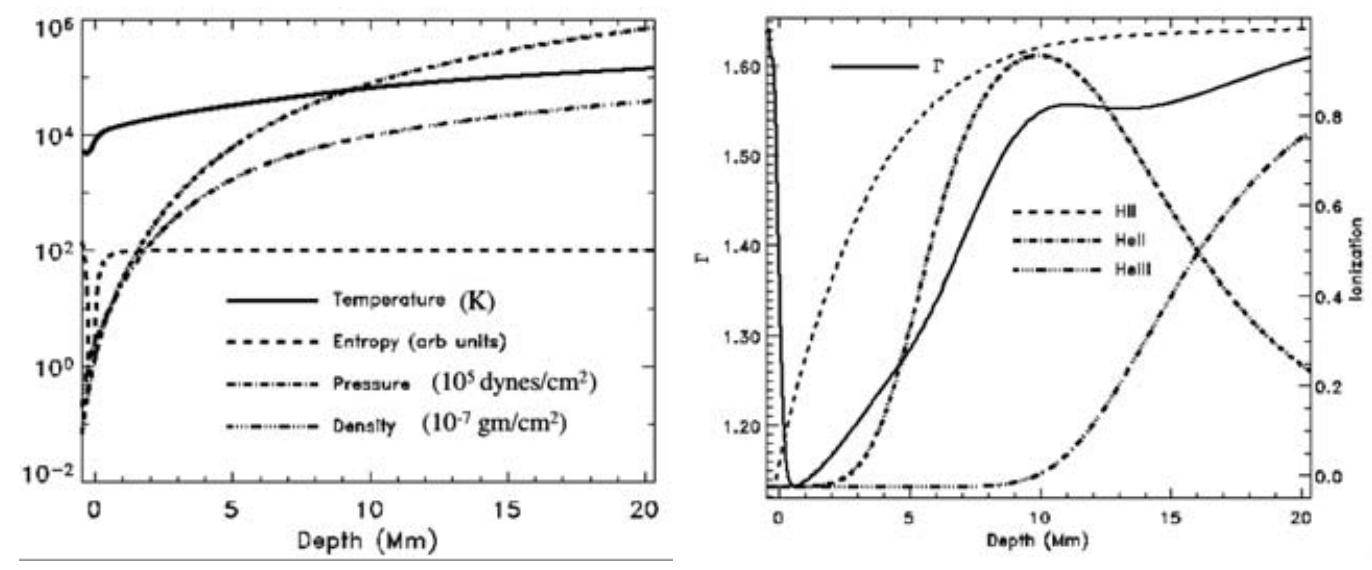

Figure 2. Mean atmosphere for $20 \mathrm{Mm}$ deep simulation.

turnover times, then another 2 turnover times at $24 \mathrm{Mm}$ wide and finally for 1.5 turnover times at $48 \mathrm{Mm}$ wide. It is thermally well relaxed but is not quite dynamically relaxed at the largest scales. At these scales it is also necessary to include the effects of rotation, so we have included f-plane rotation (constant rotation rate throughout the domain). A surface shear layer develops as observed on the Sun. We have not yet included magnetic fields but will be adding them. We use a grid of 500 cubed so the horizontal grid size is $100 \mathrm{~km}$. The vertical grid size varies from $12 \mathrm{~km}$ at the surface increasing to $70 \mathrm{~km}$ at the bottom. Spatial derivatives are calculated as sixth order finite differences on a staggered grid and time advance is by a low memory third order Runge-Kutta scheme. In order to make it realistic we calculate the pressure as a function of density and energy per unit mass from a tabular equation of state that includes LTE ionization and excitation of hydrogen, helium and other abundant elements. Radiative heating and cooling drives the convection and controls the structure of the photosphere. Since the domain includes optical depth unity neither the optically thin nor the diffusion limits are appropriate, so we solve the transfer equation assuming LTE and reduce the number of frequencies by using a multigroup method whereby the opacity and source function at each frequency is assigned to one of 4 bins according to the magnitude of the opacity (Nordlund 1982, Stein \& Nordlund 2003). Regner Trampedach is working on an alternative method which solves for the radiation field at 50 specially selected frequencies.

Figure 2 shows the mean atmosphere. The temperature goes from about $4000 \mathrm{~K}$ near the temperature minimum to a little over $100,000 \mathrm{~K}$ at the bottom. The density and pressure vary by over 6 orders of magnitude, so it is very highly stratified. It includes hydrogen ionization, first helium ionization and most of the second helium ionization zone.

Figure 3 shows a vertical slice through the domain. Red are downflows and blue upflows and fluid streamlines are shown. ¿From a movie one can see that at the surface features are evolving very rapidly, on the order of minutes, while at large depths they are evolving very slowly, on the order of hours. The downflows in the intergranular lanes are swept sideways by the upflows which are diverging because of mass conservation - most of the fluid from below can not be carried into the lower density layers above but most turnover and head back down within a scale height. The downflows are swept into larger downflows which penetrate down deeper. In addition, some of the downflows are halted as they beat their way downward against the upflows. 


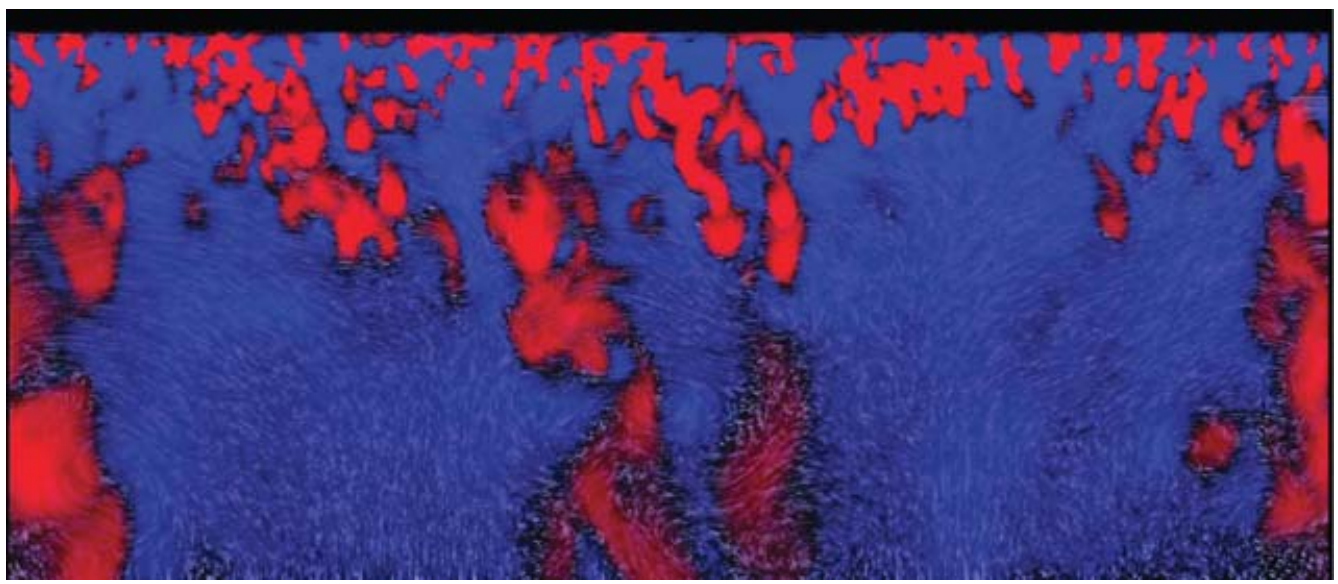

Figure 3. Fluid velocity in vertical plane. Width is $48 \mathrm{Mm}$ and depth $20 \mathrm{Mm}$. Light gray (Red) are downflows and dark gray (blue) are upflows. Streaks are streamlines. At the top of the convection zone the structures are small granules. The size of the structures increases gradually with increasing depth as the diverging upflows either halt some of the downflows or sweep them into the larger downflows.

Images of the vertical velocity in horizontal planes at different depths (Fig. 4) clearly show that the size of the horizontal structures changes continuously from granulation at the surface to supergranulation at large depths. There is no particular scale of mesogranulation and probably not of supergranulation either. Another way of visualizing this is to look at streamlines (Fig. 5). Here streamlines have been seeded uniformly at 2 $\mathrm{Mm}$ intervals at the surface. The left image shows that fluid moving up at the surface comes from very small isolated locations at depth. The right image shows that fluid moving down at the surface collects in the boundaries of supergranulation scale cells at large depth. Further, the catch basin for the fluid that reaches the bottom to form the downflow boundaries of the supergranules at large depth comes from a small portion of the surface that tends to lie approximately above the supergranule boundaries.

Figure 6 shows the velocity spectrum for the simulated vertical and horizontal velocities at a height of $200 \mathrm{~km}$ above continuum optical depth unity and for high resolution MDI data. The total velocity (solid) is separated into convective (dashed) and oscillatory (dotted) components by whether $\omega$ is less or more than $c k$ where $c$ is taken to be $6 \mathrm{~km} / \mathrm{s}$. The first thing to observe is that the spectrum is smooth. There is a maximum at granulation scale. There is no feature at mesogranulation scale. There is a small enhancement with respect to the general power law in the MDI convective component at supergranulation scales. The horizontal velocity is almost purely convective. The convective velocity is dominated by convective motions at small scales but by oscillatory motions at large scales.

\section{Application: helioseismology}

The reason these simulations are useful to helioseismology is because they posses a rich spectrum of modes that are excited naturally in the simulation (Fig. 7). As an initial step, Junwei Zhao calculated the time-distance diagram for the first 8.5 hours of the simulation and compared it with that from the MDI high resolution data which gave very similar results (Georgobiani et al. 2007). He has also looked at where the waves are produced and how they propagate by correlating each point at the surface with all the 


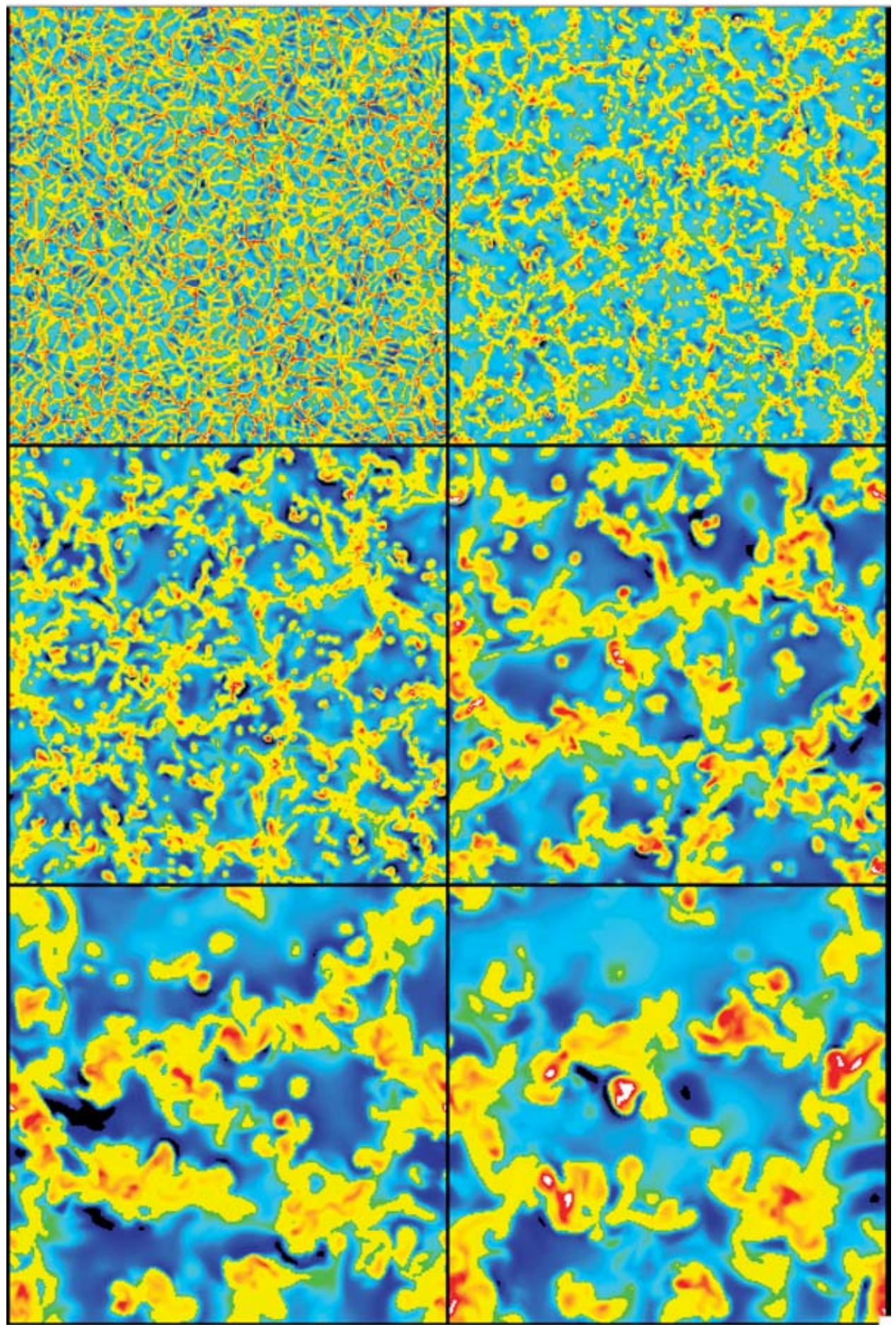

Figure 4. Vertical velocity in horizontal slices at increasing depth. Going from the surface at the top left across and down at depths of 2, 4, 8, 12 and $16 \mathrm{Mm}$. Red and yellow (light gray) are downflows, blue and green (dark gray) are upflows. Each depth is scaled separately. 


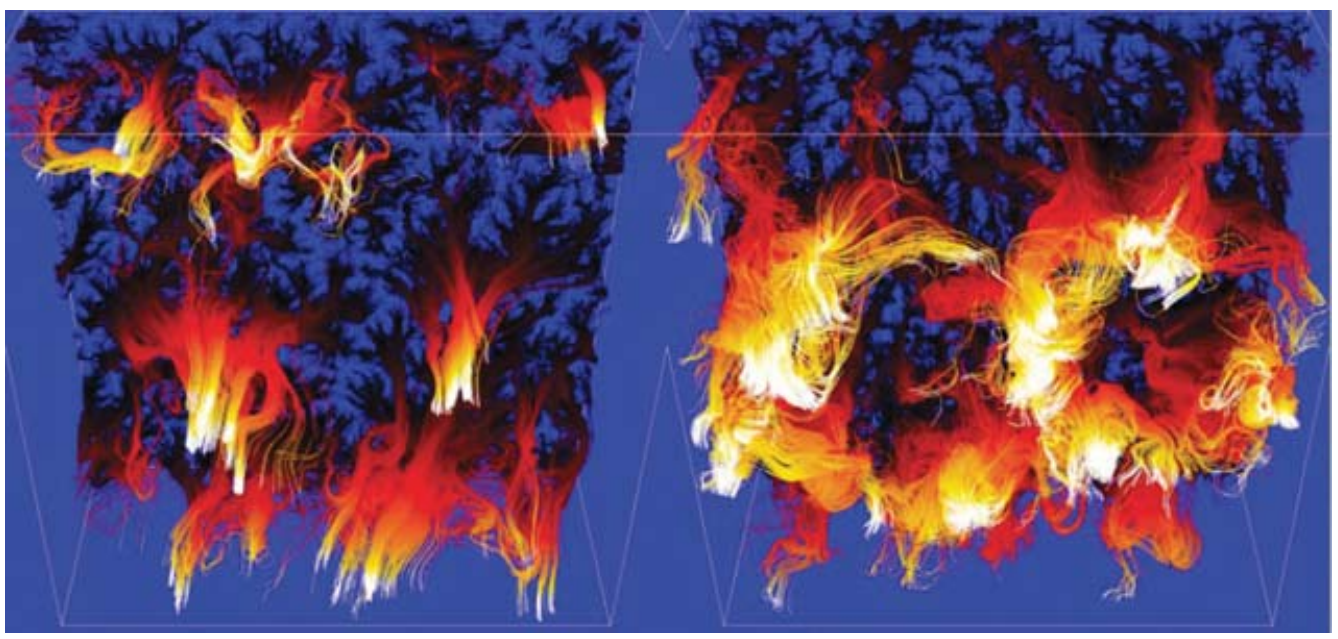

Figure 5. Streamlines seeded at $2 \mathrm{Mm}$ intervals at the surface. The color coding shows depth, with dark at the surface and white at the bottom. On the left are streamlines for fluid moving up at the surface showing that the fluid that reaches the surface originates from very small areas at large depths. On the right are streamlines for fluid moving down at the surface, showing that downflows collect in larger downflows with increasing depth becoming the boundaries of the supergranulation cells near the bottom.

points below. This essentially produces a Green's function for the waves (Fig. 8). The waves at the surface propagate into the interior and spread horizontally and some power returns to the surface.

Junwei Zhao and Aaron Birch have also compared the results from a time-distance analysis with what is actually in the simulation. Figure 9 shows the travel time difference between north and south traveling waves and the simulated north-south velocity at 200 $\mathrm{km}$ up in the photosphere. The patterns are similar but not identical. Also, the quality of the agreement is different for the north-south and east-west directions. The same issue has occurred in analyzing the MDI data. Clearly, more work needs to be done.

These data are available to the community at the Stanford Helioseismology Archive (http://sha.stanford.edu/).

\section{Wave propagation}

To understand local helioseismology and chromospheric and coronal dynamics and heating it is also important to study wave propagation. Viggo Hansteen has studied MHD wave propagation and mode coupling in $2 \mathrm{D}$ using a similar code. There is a lot of mode coupling and reflection that occurs at $\beta=1$ surface, where $\beta$ is the ratio of gas to magnetic pressure. However, it is not easy to separate the different wave modes in such non-linear calculations.

Idealized linear wave propagation studies are also important. They are much faster than realistic non-linear calculations. They can be used to study many different cases, to explore parameter space and to isolate different physical effects. I present a few of many examples.

Joe Werne has shown that the difference in wave travel times in opposite directions along the ray path can indeed be inverted to find the fluid flow. In a $2 \mathrm{D}$ experiment, he analyzed waves produced by sources at random locations and times in the presence of a large scale flow. He calculated the acoustic travel times in one direction and its opposite 


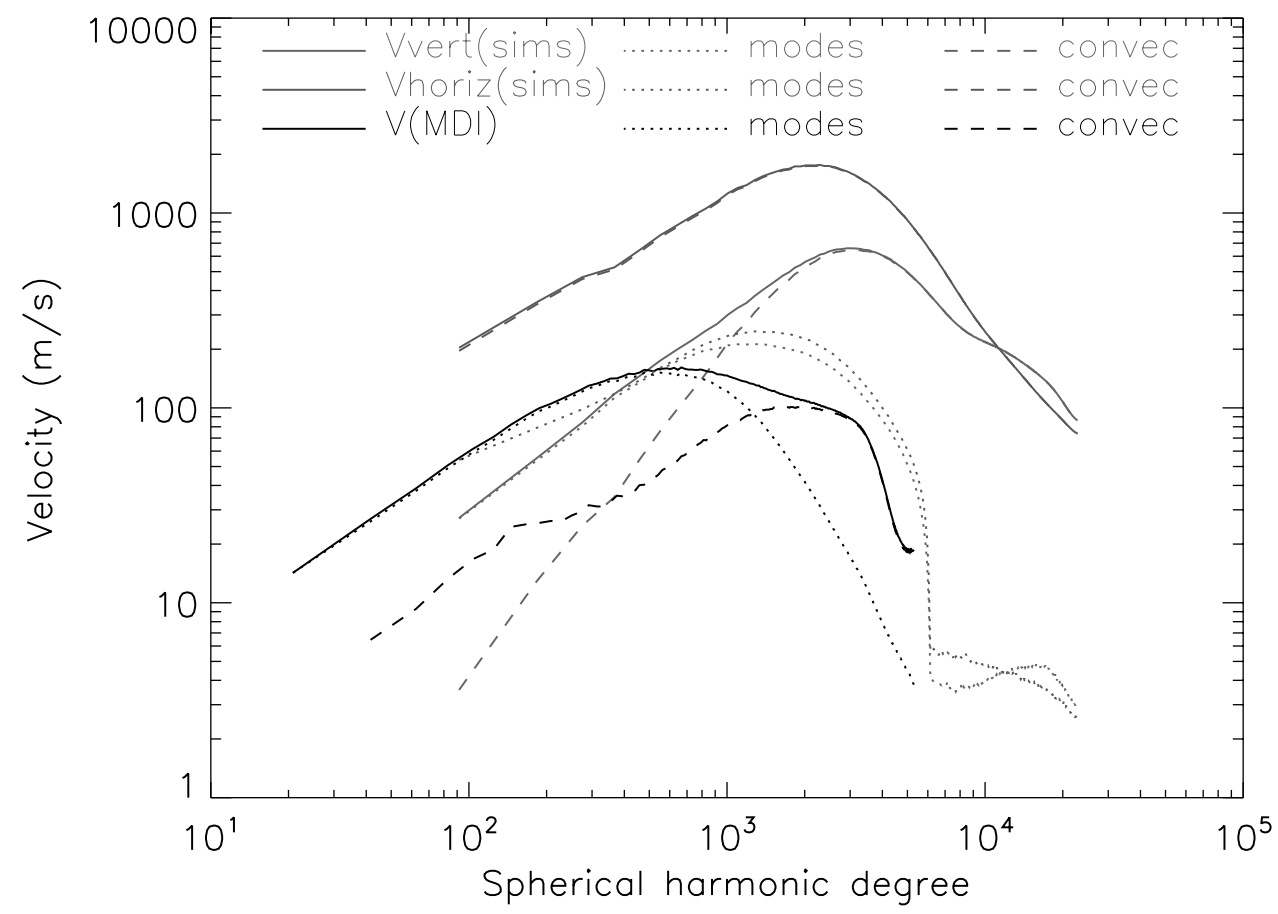

Figure 6. Velocity spectra for the simulation and MDI observations, separated into convective (dashed lines), oscillatory (dotted lines) and total (solid lines) components. The dark or red set is for the MDI high resolution velocity observations. The light or green set is for the vertical simulation velocities. The medium grey or blue set is for the horizontal simulation velocities. The oscillatory signal is negligible in the horizontal velocity but dominates the vertical velocity and MDI Doppler velocity on large scales $(\ell \leqslant 1000)$. The horizontal velocity is almost purely convective.

and then their difference and showed it was possible to recover the large scale flow field properties.

Paul Cally and Hannah Schunker studied wave transmission and mode coupling at Alfven speed (a) equals the sound speed (c) (Schunker \& Cally 2006). For acoustic waves propagating in the direction of the magnetic field, most of the energy gets transmitted as an acoustic slow wave propagating along the field and some gets converted to a magnetic fast wave which eventually gets refracted back down and when it again traverses the $\mathrm{a}=\mathrm{c}$ level it is mostly reconverted to an acoustic fast wave with a little energy transmitted into a magnetic (slow) wave because of the large angle of attack with respect to the magnetic field (Fig. 10). It is a very different story when an acoustic wave encounters the $\beta=1$ level at a large angle to the magnetic field (Fig. 11). In this case, most of the power gets converted into a magnetic (fast) wave, with only a little transmitted 9as an acoustic (slow) wave. The magnetic fast wave is again refracted back down, but now encounters the $\mathrm{a}=\mathrm{c}$ level propagating nearly along the magnetic field so there is not much mode coupling and it passes through as an acoustic fast wave. These types of studies are important for understanding mode coupling at the $\mathrm{a}=\mathrm{c}$ surface.

The magnetic field also reduces the acoustic cutoff frequency. Both mode coupling and the reduced acoustic frequency in the presence of strong magnetic fields allow long period acoustic waves to reach the chromosphere. Hence, there are "magnetic holes" where there is enhanced acoustic heating in the chromosphere as well as magnetic heating. Stuart 

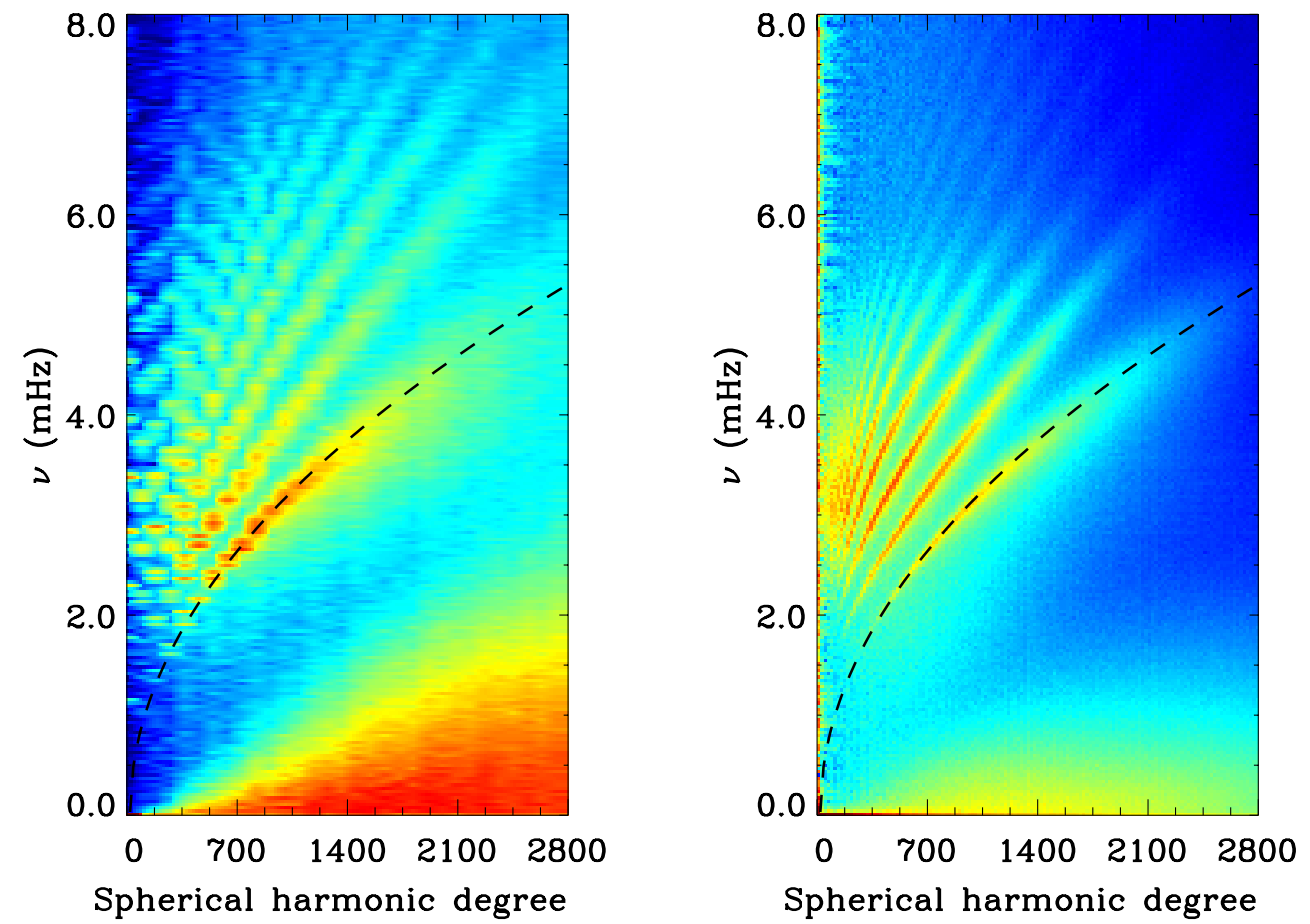

Figure 7. The power spectra ( $\ell-\nu$ diagrams) for the simulated vertical velocity (left) and the Doppler velocity from the MDI high-resolution observations (right). The dark curve represents the theoretical $f$-mode.

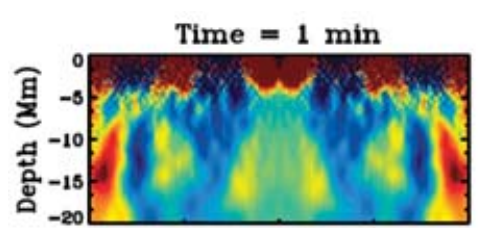

Time $=7 \mathrm{~min}$

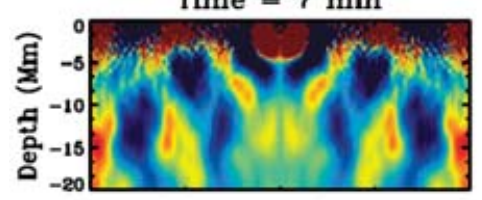

Time $=13 \mathrm{~min}$

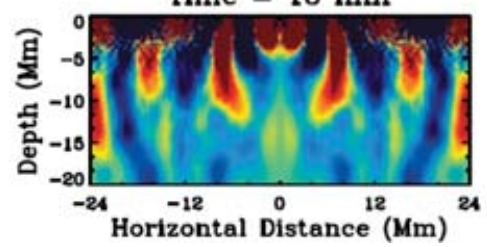

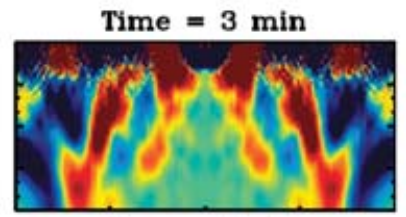

Time $=9 \mathrm{~min}$

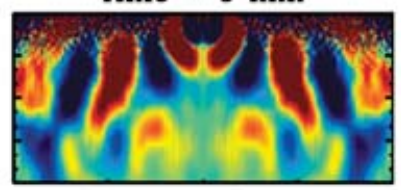

Time $=15 \mathrm{~min}$

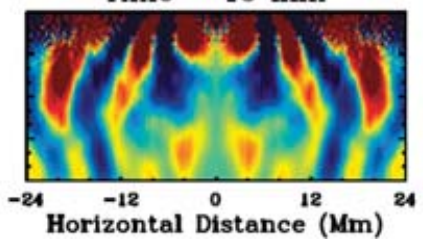

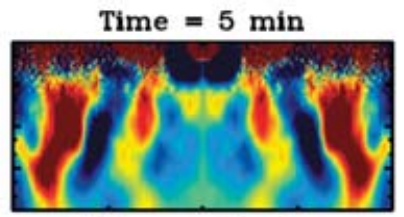

Time $=11 \mathrm{~min}$

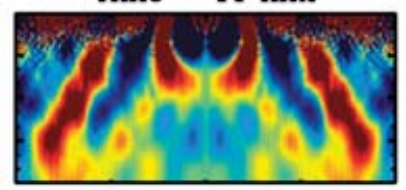

Time $=17 \mathrm{~min}$

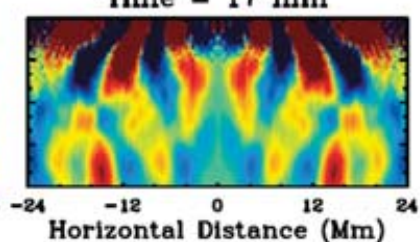

Figure 8. Wave propagation in the simulation. This diagram is obtained by computing the cross correlation of each point on the surface with all the points at a given depth and time delay and displaying the result as a function of horizontal displacement and depth for each time delay. This shows how the waves at the surface propagate into the interior and spread horizontally. 


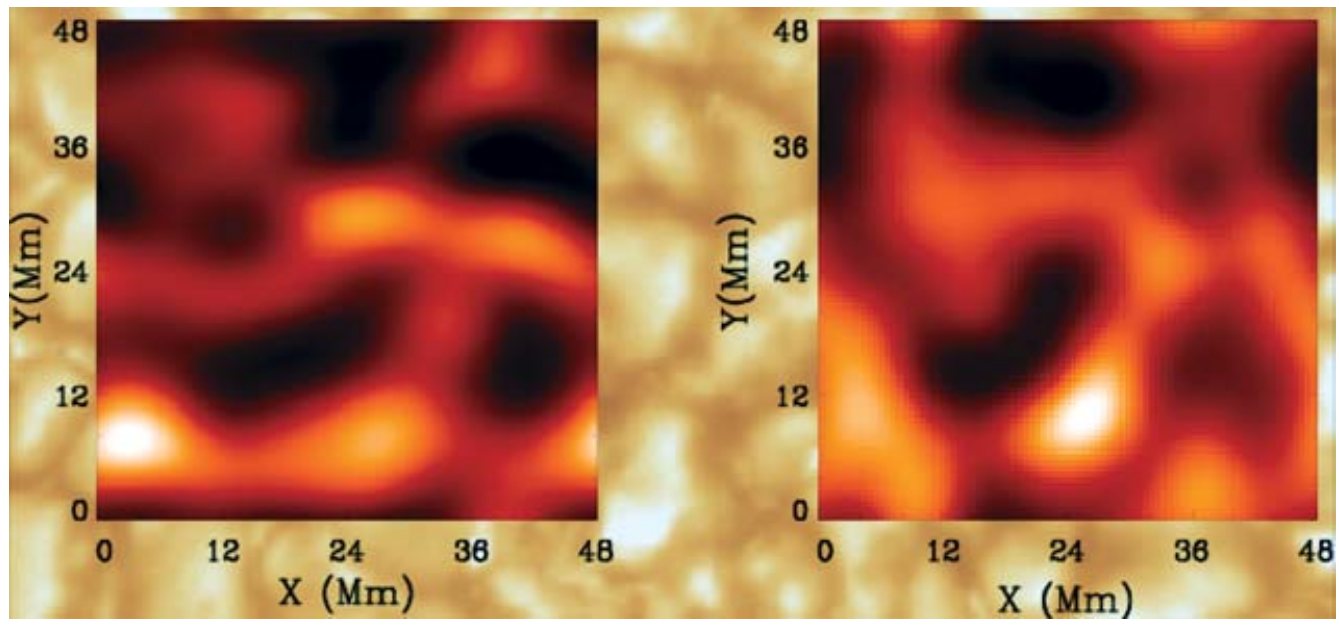

Figure 9. Comparison between the simulated horizontal velocity $\left(V_{y}\right.$, left, light is north-bound and dark is south-bound) and $f$-mode north-south travel time difference map (right, light represents faster north-bound and dark represents faster south-bound waves). A low-pass filtering has been performed to reveal the large structures.

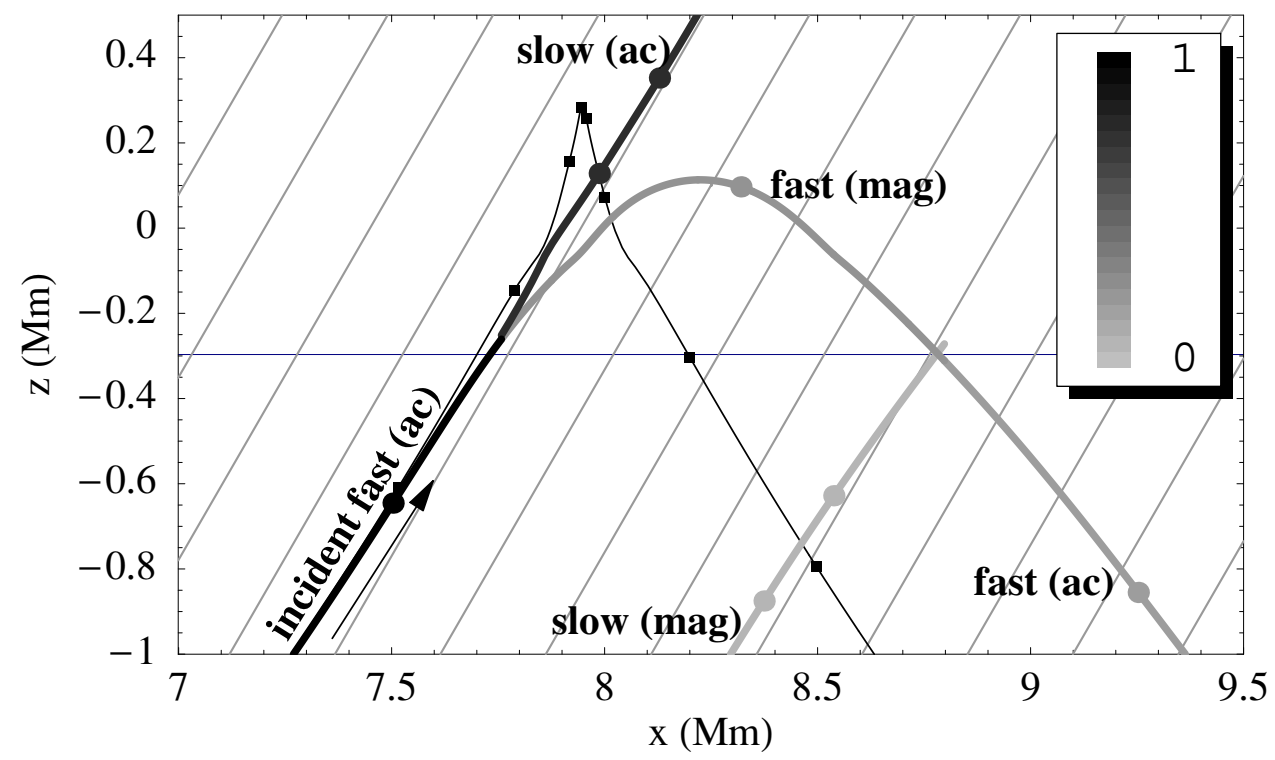

Figure 10. Wave ray paths with color coding showing the fraction transmitted for the case of an incident acoustic wave traveling nearly parallel to the magnetic field direction. The black line shows the behavior of an acoustic wave in a field free environment.

Jefferies (Jefferies et al. 2006) finds that there is more energy reaching the chromosphere as long period acoustic waves than high frequency waves where the magnetic field is strong, so locally there can be significant acoustic heating in magnetic concentrations in the chromosphere (Fig. 12).

In conclusion, simulations have much to offer in helping us test and refine local helioseismic methods, in understanding mode excitation and damping, in clarifying the dynamics and heating mechanisms in the chromosphere and corona. 


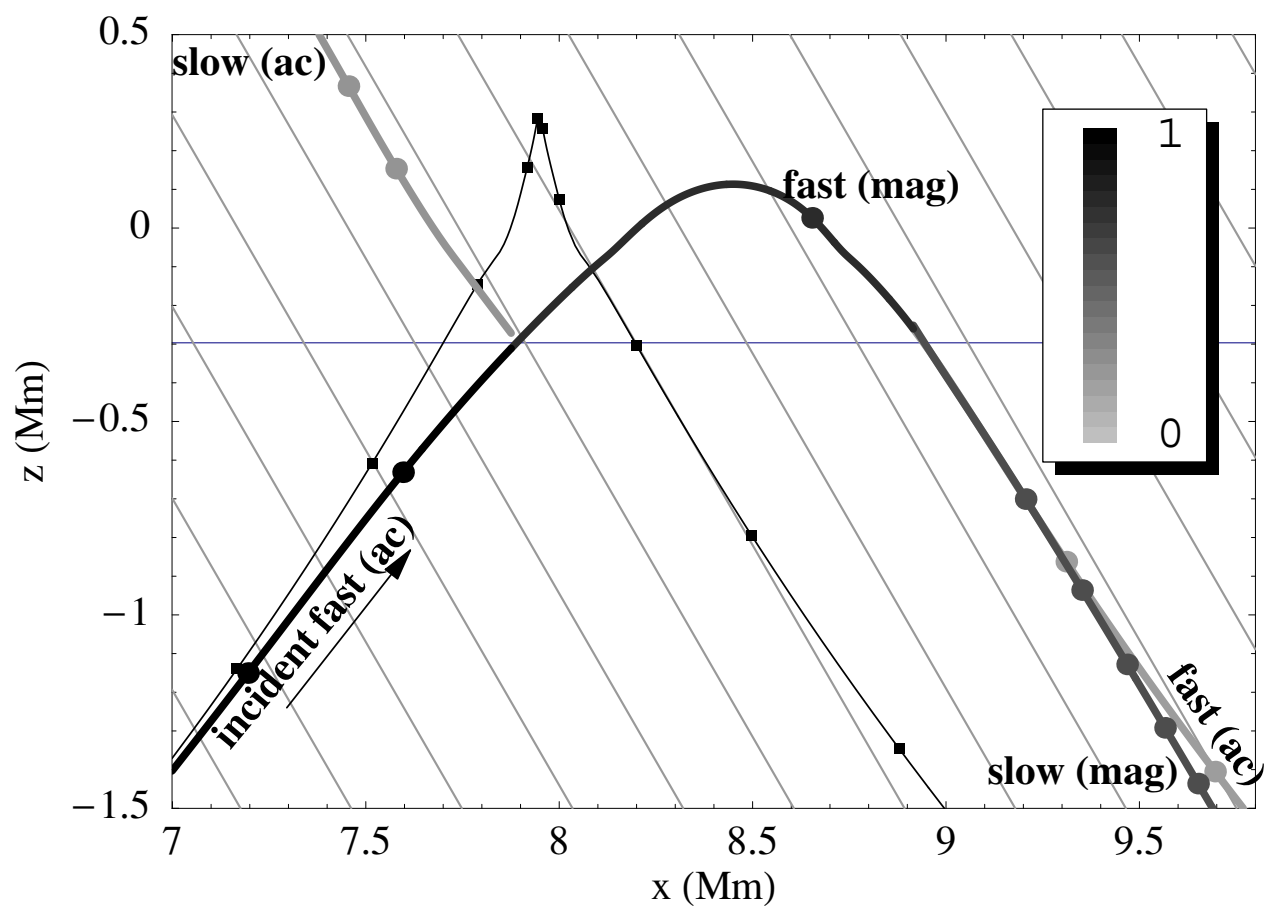

Figure 11. Wave ray paths with color coding showing the fraction transmitted for the case of an incident acoustic wave traveling nearly perpendicular to the magnetic field direction. The black line shows the behavior of an acoustic wave in a field free environment.

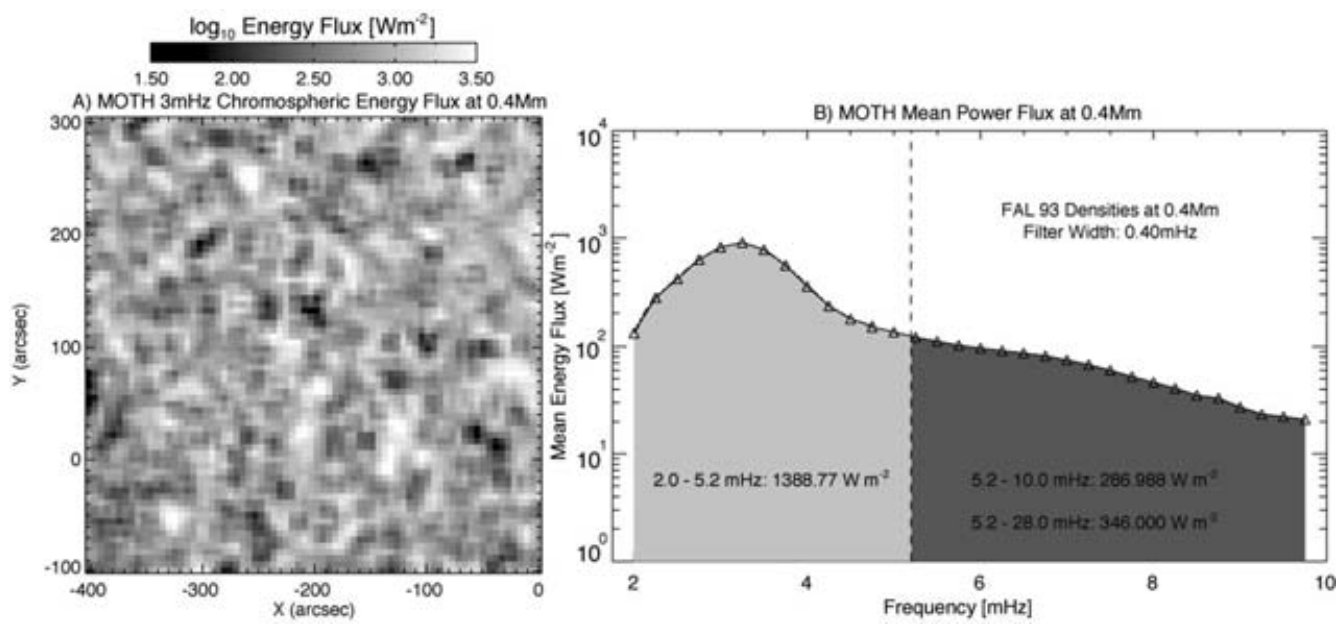

Figure 12. Wave energy flux at $3 \mathrm{mHz}$ at a height of $400 \mathrm{~km}$ above $\tau_{500}=1$. Left shows this low frequency acoustic energy flux is highly localized in space. Right shows that there is a larger acoustic flux at low frequencies than at high frequencies reaching this height.

\section{Acknowledgements}

This work was supported by NASA grants NNG04GB92G and NAG 512450 and NSF grants AST-0205500 and AST-0605738. 


\section{References}

Georgobiani, D., Zhao, J., Kosovichev, A., Benson, D., Stein, R. F., \& Nordlund, Å. 2007, ApJ (in press)

Goldreich, P., Murray, N., \& Kumar, P. 1994, ApJ 424, 466

Jefferies, S. M., McIntosh, S. W., Armstrong, J. D., Bogdan, T. J., Cacciani, A., \& Fleck, B. 2006, ApJl 648, L151

Nordlund, A. 1982, A. \& A. 107, 1

Nordlund, A. \& Stein, R. F. 2001, ApJ 546, 576

Samadi, R. \& Goupil, M.-J. 2001, A \& A 370, 136

Samadi, R., Goupil, M.-J., \& Lebreton, Y. 2001, A \& A 370, 147

Samadi, R., Nordlund, Å., Stein, R. F., Goupil, M. J., \& Roxburgh, I. 2003, A. \& A. 404, 1129

Schunker, H. \& Cally, P. S. 2006, MNRAS 1003

Stein, R., Georgobiani, D., Trampedach, R., Ludwig, H.-G., \& Nordlund, Å. 2004, Sol. Phys. 220, 229

Stein, R. F. \& Nordlund, Å. 2001, ApJ 546, 585

Stein, R. F. \& Nordlund, Å. 2003, in I. Hubeny, D. Mihalas,, K. Werner (eds.), ASP Conf. Ser. 288: Stellar Atmosphere Modeling, 519

\section{Discussion}

J. Christensen-DalsgaArD: With your marvellous $48 \mathrm{Mm}$ simulations you should be able to extract accurate frequencies of the modes excited in the simulation. Have you compared these with corresponding frequencies of a mean model?

STEIN: We have not made a comparison with the frequencies in the mean model, only with the MDI observations.

M. Steffen: 1. What do you mean by quenching? 2. What are your ideas when putting magnetic fields into your big simulations?

STEIN: 1. By quenching we refer to a procedure in which we scale the diffusive flux by the ratio of the third to first differences of the variable diffused. This reduces the diffusion on scales larger than six grid zones and increases it on smaller scales. 2. We intend to add magnetic fields to the supergranulation scale model in two ways: First, by extrapolating an observed active region surface magnetic field into the interior and letting it evolve under the influence of the convective motions. This is desired by the local helioseismologists to validate their analysis methods. Second, by advecting horizontal field into the domain by inflows (upflows) at the bottom. This will allow us to study the emergence of flux in the quiet Sun and the formation and maintenance of the magnetic network.

M. GoupiL: Can you quantify the improvement you obtain when not taking out the eigenfunction form the source function and what is the main consequence?

Stein: We compared the eigenfunctions of the modes in our $6 \mathrm{Mm}$ wide simulation with those calculated by Christensen-Dalsgaard and found them almost identical except in the upper photosphere. You can see the comparison in Stein \& Nordlund 2001. 